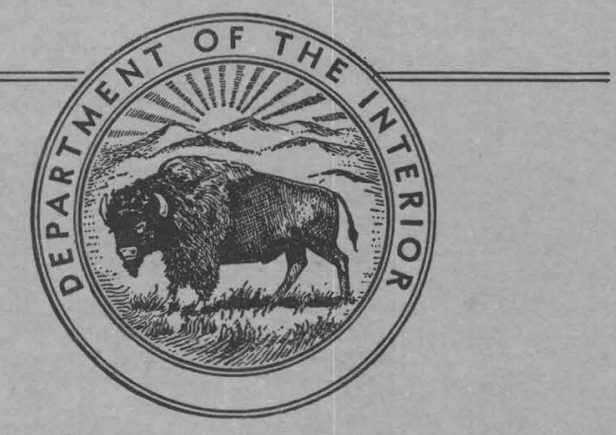

November 1949

\title{
AVERAGE ANNUAL RUNOFF IN THE WIND RIVER BASIN IN WYOMING
}

By

Roy E. Oltman and Hubert J. Tracy 


\section{UNITED STATES DEPARTMENT OF THE INTERIOR \\ J. A. Krug, Secretary GE OLOGICA L S UR V E Y \\ W. E. Wrather, Director}

WASHINGTON, D. C.

Free on application to the Director, Geological Survey, Washington 25, D. C. 


\section{AVERAGE ANNUAL RUNOFF IN THE WIND RIVER BASIN IN WYOMING}

By

Roy E. Oltman and Hubert J. Tracy.

CONTENTS

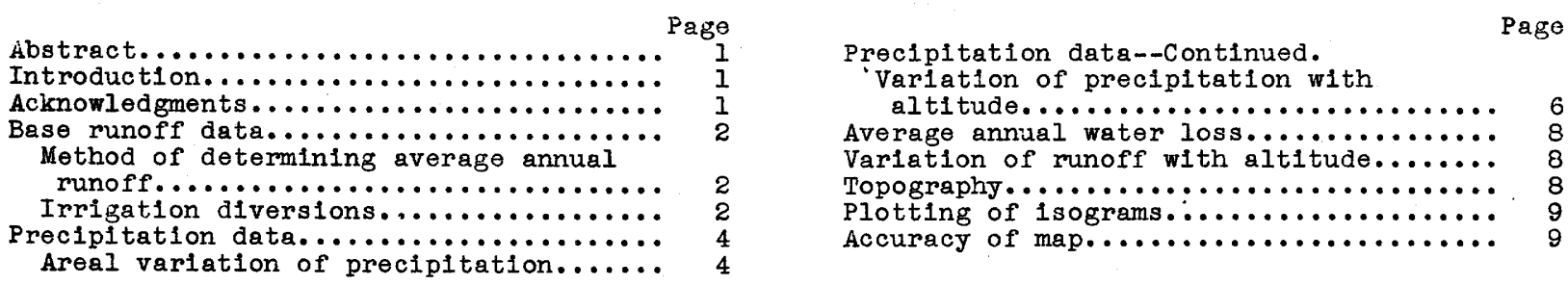

ILLUSTRATIONS

Plete 1. Pverage annugl Page

Plate 1. Average annual runoff in the wind River Basin...................... In pocket

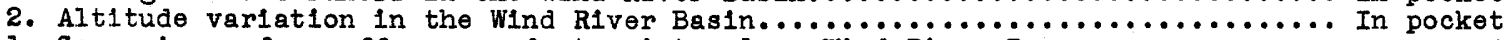

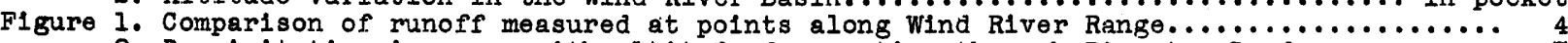

2. Precipitation increase with altitude for section through Riverton-Iander.......... 7

3. Comparison of preclpitation-altitude graphs for sections of Wind River Range....... 8

4. Composite average annual loss varlation with altitude along north side Wind River

Range.................................................... 8

TABLES

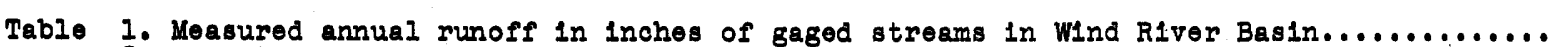

2. Annual precipitation records of stations in or adjacent to Wind River Basin........

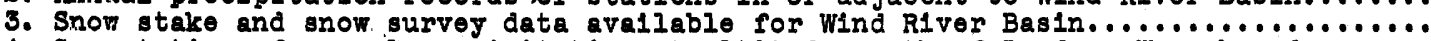

4. Computation of annual procipltation at altitude south of Lander, Wyo. based on

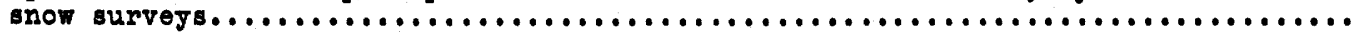

\section{ABSTRACT}

Average annual runoff from the mountain ranges bordering the Wind River Basin is large In amount and comparatively stable; average annual minoff from the arid plains region of the basin 1s very low and there are no perennlal streams having their source in this region. A map of the basin was propared that show the distribution of average annual munoff in inches by means of 1 sograms. The construction of the map was based on avallable streamflow records, climatolog 10 records, and a developed precipitation-altitude relationship for the Wind RIvor Mountains. Location of the 1.sograms in the arid portion of the basin was based malnly on personal observation made on fleld reconnalesance of the basin.

\section{INTRODUCTION}

The constmuction of a map showing average annual runoff in the Wind River Basin $1 \mathrm{~s}$ made difflcult by the lack of sufflolent base data and the wide range of munoff within the basin. More than half of the basin has an average annual precipitation of less than 10 inches, and the resulting munoff is very low. In contrast, the area along the Wind R1ver and OwI Creok Mountains recelves heavy precipitation, the amount varying with altitude. Climatic data, wh th the exception of a few snow surveys along the flanks of the Wind River Range, are limlted to records taken at a fow points of low altitude in the valloy. Stroam-gaging station records of prime usefulness in delinoating a monof map in the basin are limited to points along the foot of the Wind River Range with the exception of a few recently ostablished stations in the arid part of the basin. A munoff map of the WIni River Basin was constructed by making use of the limited stream-flow and climatic data with dus regard to hydrologic principles. Tho ostimates of munoff from ungaged arid areas were based partiy on data noted on a fleld reconnalssance.

\section{ACKNOWLEDGMBHTS}

This report was complled, as part of the program of the Interlor Department for development of the Missourl River Basin, hy the taff of the Lincoln Reglonal Fleld Office, Special Reports and Investigations section of the Surface Water Branch, J. V. B. Wells, Chlef.

Indebtednes to the following persons or offices for the services listed is gratefully 
acknowledged:

District Office, Surface Water Branch, Denver, Colo., furmished gaging station records in advance of publication.

Mr. Henry W. Davis, Hydrographer, Bureau of Indian Affalrs, furnished many unpublished gaging station records and gave valuable advice on the magnitude of irrigation diversions.

Mr. K. R. Melin, Technical Coordination Branch, Geological Survey, guided the authors on a l-week trip through the basin and furnished helpful advice in estimating the munoff from arid parts of the basin.

Riverton Project Office, Bureau of Reclamation, furnished figures on annual diversions from the Wind River into the Wyoming Canal from 1926-48.

U. S. Weather Bureau Offices at Chejenne, Wyo. and Kansas C1ty, Mo., furnished computed normal water-content figures for the snow stakes operated by the Weather Bureau and the precipitation records for. Oregon Trail CrossIng and T-Cross Ranch stations.

\section{BASE RUNOFF DATA}

Many of the streams in the Wind River Basin have been gaged for a perlod of several years by the U. S. Geological Survey and the Bureau of Indian Affairs. The distribution of gaging stations is such (and. this has been dictated by the need for information on 1rrigation supplies) that a fairly accurate knowledge of the annual runoff from streams draining the wind River Mountain Range bordering the basin can be determined, but the munoff from the arid plains area of the basin must be estimated on the basis of past experience and climatic data. The locations of gaging stations used in this study are shown on plate 1 , general map of the basin upon which the runoff isograms are plotted. Table 1 contains a list of annual runoff depths in inches for different streams of the basin that have been gaged at some time during the period 1918-48. The munoff in inches has been computed from the latest published drainage area figures; these are listed in the box with each stream name in table 1 . Those gaging station records that are essentially records of net flow after substantial irrigation diversions have been omitted from table 1. Many of the annual runoff values listed in table 1 are partialiy estimated to complete the water year for some records at gages not operated during the winter months or to correct for unknown storage changes in reservolrs above the gaging station. Such partially estimated values are footnoted.

\section{Method of Determining Average Annual Runoff}

All the streams issuing from the Wind River Range show a marked similarity in runoff characterlstics. Figure 1, a plot of the annual munoff values for all the gaged streams along the wind River Range from 1918 to 1948 , shows the generally good relationship of annual munoff measured at one station to that measured at other stations.
Because of thelr sparseness, all existing records of runoff were utilized. "Averages" ilsted in table 1 are the arithmetical averages of the figures in the column above.

The available munoff records at many of the stations listed in table 1 are for a short perlod; because of year-to-year varlations in the munoff from any drainage area, it was necessary to adjust the short-term records to a long-term base. The perlod of years from 1918 to 1948 was used as the base. The lower gaging station on Bull Lake Creek was selected as the index station for runoff from the Wind River Range; because of the complete record from 1918 to 1948 , and because of the central location of 1 ts drainage area along the range. The ratio of the annual runoff to the average for this station for the period 1918-48 was computed for each year and listed in the second colum of table 1 under "index percent". These percentages were used to adjust the munoff from the other gaged areas for each year of record to make them comparable. This was done by dividing the listed annual munoff by the 11sted percentage for each year of minoff record and then taking the average of these quotients; the "adjusted average" is listed beneath the "average" in table 1.

The adjusted average runoff thus determined was corrected for Irrigation diversions (as explained in the next section of this report) where these were sizeable, and the corrected averages were used in the construction of the runoff map. The map is based, therefore, on virgin flow; large return flows from irrigation, as in Fivemile Creek, were not considered.

The records for the streams issulng from the Wind River Range were adjusted as described above; the records for the streams in the other portions of the Wind River Basin were adjusted as described under "Precipitation Data" later in this report.

\section{Irrigation Diversions}

Accurate information on the quantity of irrigation diversions from streams in the Wind River Basin is not available for each year of the period 1918-48. The total number of acres irrigated in each major sub-basin can be obtained from Bureau of the Census publications for the years ending 1919, 1929, and 1939 , and were given considerable weight in estimating probable net diversions above rach gage in arriving at the irrigation adjustment listed in table 1. For many streams the probable net diversion was very small and was Ilsted as zero. However, a few hundred acres may have been irrigated above the gaging station on such streams, but the crop ralsed was generally hay, and much of the applied water became return flow above the gage. The irrigation adjustment on a few of the streams listed in table 1 was estimated by noting the probable capacity of the one by-pass irrigation ditch. In general, the irrigation adjustments listed in tabie 1 represent a small proportion of the total munoff of the stream; any inaccuracy in the estimated adjustment will not make the adjusted runoff unreliable. 


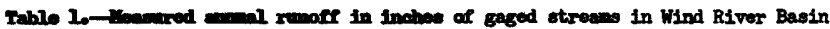

\begin{tabular}{|c|c|c|c|c|c|c|c|c|c|c|c|c|c|c|c|c|c|c|}
\hline \multirow[b]{2}{*}{ moter } & \multirow[b]{2}{*}{$\begin{array}{c}\text { Index } \\
\text { peinomit } \\
\end{array}$} & \multicolumn{17}{|c|}{ Stroean and drainage area } \\
\hline & & 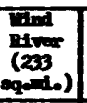 & 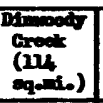 & 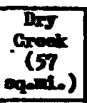 & 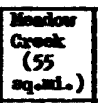 & 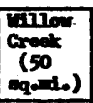 & 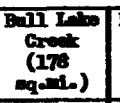 & 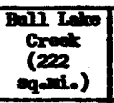 & 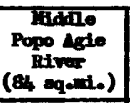 & 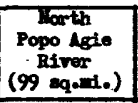 & $\begin{array}{c}\text { Horth } \\
\text { Popo Agic } \\
\text { River } \\
(140 \text { sq.mi.) })\end{array}$ & 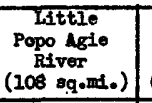 & $\begin{array}{c}\text { Ifttile } \\
\text { Popo Agie } \\
\text { R1 ver } \\
\text { (331 sq.ani.) }\end{array}$ & $\begin{array}{c}\text { Little } \\
\text { Wind } \\
\text { River } \\
\text { (118 sq.mi.) }\end{array}$ & \begin{tabular}{|l|} 
North Fork \\
Little wind \\
River \\
(127 sq-mi.) \\
\end{tabular} & \begin{tabular}{|l|} 
Beaver \\
Creek \\
(11l8 \\
sq.mif.) \\
\end{tabular} & $\begin{array}{c}\text { Bactoter } \\
\text { Creak } \\
\text { (17.78 } \\
\text { sq.mi.) } \\
\end{array}$ & 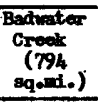 \\
\hline $\begin{array}{l}1918 \\
1999 \\
1920\end{array}$ & $\begin{array}{r}119 \\
72 \\
118\end{array}$ & & $\begin{array}{r}-22.6 \\
17.6 \\
18.8\end{array}$ & & & & & $\begin{array}{l}21.3 \\
12.9 \\
2.0\end{array}$ & $\begin{array}{r}15.08 \\
9.5 \\
19.8\end{array}$ & & & & & & & & & \\
\hline $\begin{array}{l}1924 \\
1922 \\
1923 \\
1924 \\
1925\end{array}$ & $\begin{array}{l}135 \\
136 \\
131 \\
122 \\
118\end{array}$ & & $\begin{array}{l}21.6 \\
22.4 \\
22.7 \\
2.1 \\
21.2\end{array}$ & $1 \mathbf{6} 2$ & $\begin{array}{l}: 2.9 \\
: 3.7\end{array}$ & 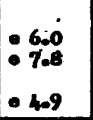 & & $\begin{array}{l}24.0 \\
2120 \\
23-4 \\
2126 \\
21.0\end{array}$ & 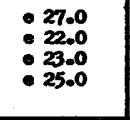 & & & & & $\begin{array}{l}20.0 \\
18.9 \\
18.9 \\
20.7 \\
16.5\end{array}$ & $\begin{array}{l}18.0 \\
14.5 \\
17.3 \\
21.9 \\
14.4 \\
\end{array}$ & & & \\
\hline \begin{tabular}{l|}
1926 \\
1927 \\
1928 \\
19293 \\
1930
\end{tabular} & $\begin{array}{c}96 \\
126 \\
121 \\
80 \\
112\end{array}$ & & $\begin{array}{l}18.9 \\
19.2 \\
19.7 \\
16.3\end{array}$ & $\begin{array}{c}11.2 \\
12.7 \\
12.8 \\
8.4 \\
11.2\end{array}$ & & $\begin{array}{l}0400 \\
: 5.5 \\
66.9 \\
64.3 \\
6.2\end{array}$ & & $\begin{array}{l}17.1 \\
22.4 \\
21.5 \\
14.5 \\
20.0\end{array}$ & & & & & & $\begin{array}{l}15.2 \\
16.7 \\
16.2 \\
111.6 \\
11.0\end{array}$ & $\begin{array}{r}12.8 \\
15.4 \\
16.0 \\
9.4 \\
12.6\end{array}$ & & & - \\
\hline $\begin{array}{l}1931 \\
1932 \\
1933 \\
1994 \\
1935\end{array}$ & $\begin{array}{c}68 \\
108 \\
104 \\
61 \\
93\end{array}$ & & & $\begin{array}{r}10.0 \\
11.0 \\
11.0 \\
5.00 \\
9.8 \\
\end{array}$ & & $\begin{array}{l}06.3 \\
: 5.4 \\
: 5.1 \\
01.9 \\
0.3 \\
\end{array}$ & & $\begin{array}{l}12.2 \\
18.4 \\
18.6 \\
10.9 \\
16.6\end{array}$ & & & & & & $\begin{array}{r}8.4 \\
13.6 \\
\mathbf{1 4 . 0} \\
7.3 \\
16.1\end{array}$ & $\begin{array}{r}8.4 \\
12.1 \\
11.0 \\
6.3 \\
14.4\end{array}$ & & & \\
\hline $\begin{array}{l}1936 \\
1997 \\
1993 \\
1999 \\
1940\end{array}$ & $\begin{array}{r}94 \\
103 \\
76 \\
64 \\
52\end{array}$ & & & $\begin{array}{r}9.4 \\
0 \quad 8.5 \\
010.1 \\
0 \quad 7.2 \\
04.3\end{array}$ & & 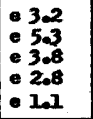 & & $\begin{array}{r}16.8 \\
0.18 .4 \\
13.5 \\
11.4 \\
9.2\end{array}$ & & & $\begin{array}{l}5.4 \\
2.9\end{array}$ & & $\begin{array}{l}2.1 \\
1.0\end{array}$ & $\begin{array}{r}\mathrm{H}_{4.8} \\
15.2\end{array}$ & $\begin{array}{l}10.6 \\
12.0\end{array}$ & $\begin{array}{r}1.1 \\
0.8\end{array}$ & & \\
\hline $\begin{array}{l}1941 \\
1942 \\
1943 \\
2946 \\
1945\end{array}$ & $\begin{array}{r}85 \\
91 \\
127 \\
102 \\
94 \\
\end{array}$ & & & $\begin{array}{l}10.2 \\
10.4 \\
12.0 \\
13.1 \\
111.6\end{array}$ & $\begin{array}{l}-3.4 \\
\text { e } 2.7 \\
\text { e } 3.7 \\
0400 \\
03.2 \\
\end{array}$ & $\begin{array}{l}\text { e } 6.0 \\
\text { e } 5.5 \\
\text { e } 7.4 \\
=8.0 \\
\quad 5.6\end{array}$ & $\begin{array}{l}21.0 \\
27.9 \\
23.7 \\
22.8\end{array}$ & $\begin{array}{l}15.1 \\
16.2 \\
22.7 \\
18.2 \\
16.7 \\
\end{array}$ & & & $\begin{array}{r}8.5 \\
9.8 \\
15.0 \\
13.0 \\
12.0 \\
\end{array}$ & & $\begin{array}{r}3.4 \\
3.0 \\
4.4 \\
4.5 \\
4.5 \\
\end{array}$ & & $\begin{array}{l}\text { e } 11.6 \\
\text { e } 12.5 \\
\text { e } 18.5 \\
\text { e } 17.6 \\
\text { e } 14.2\end{array}$ & 1.9 & & \\
\hline $\begin{array}{l}1946 \\
1947 \\
1948\end{array}$ & $\begin{array}{r}75 \\
130 \\
94\end{array}$ & $\begin{array}{l}9.1 \\
11.6 \\
104\end{array}$ & & $\begin{array}{r}8.5 \\
-16.0 \\
-13.2 \\
\end{array}$ & $\begin{array}{l}\text { e } 2.2 \\
\text { e } 3.5 \\
\text { e } 3.3 \\
\end{array}$ & $\begin{array}{l}e 3.4 \\
e \\
e \\
e .408 \\
\end{array}$ & $\begin{array}{l}18.1 \\
28.0 \\
20.6\end{array}$ & $\begin{array}{l}13.3 \\
23.2 \\
16.8 \\
\end{array}$ & & $\begin{array}{l}14.0 \\
22.0 \\
12.9\end{array}$ & $\begin{array}{r}8.3 \\
74.9 \\
7.2 \\
\end{array}$ & $\begin{array}{r}14.3 \\
8.1 \\
\end{array}$ & $\begin{array}{l}2.9 \\
6.1 \\
3.1\end{array}$ & & $\begin{array}{l}12.5 \\
\text { e } 18.8 \\
\text { e } 11.9\end{array}$ & & $\begin{array}{l}0.5 \\
0.7 \\
0.4\end{array}$ & 0.4 \\
\hline Anerage & & 10,4 & 20.1 & 10.5 & 3.3 & 5.1. & 232 & 17.8 & 20.3 & 16.3 & 9.7 & 21.2 & 3.5 & 15.2 & 13.8 & 1.3 & 0.5 & 0.4 \\
\hline arjusted average & & 10.7 & 1850 & nol & 3.1 & 5.2 & 23.0 & 17.88 & 16.8 & 16.4 & 10.2 & 9.8 & 3.7 & 34.03 & . 13.2 & 1.8 & 0.5 & 0.5 \\
\hline Irrigation adjastinent & & 0.2 & 0.0 & 0.0 & 0.0 & 0.0 & 0,0 & 0.0 & 0.0 & 0.0 & 1.5 & 0.0 & 1.5 & 1.5 & 0.8 & 0.2 & 0.1 & 0.1 \\
\hline Adfusted for irrigation t? & & 10.8 & 18.0 & 11.1 & 3.2 & 5.2 & 23.0 & 17.8 & 16.8 & 16.4 & 212.7 & 9.8 & 4.9 & 15.8 & 14.0 & 2.0 & 0.6 & 0.6 \\
\hline
\end{tabular}




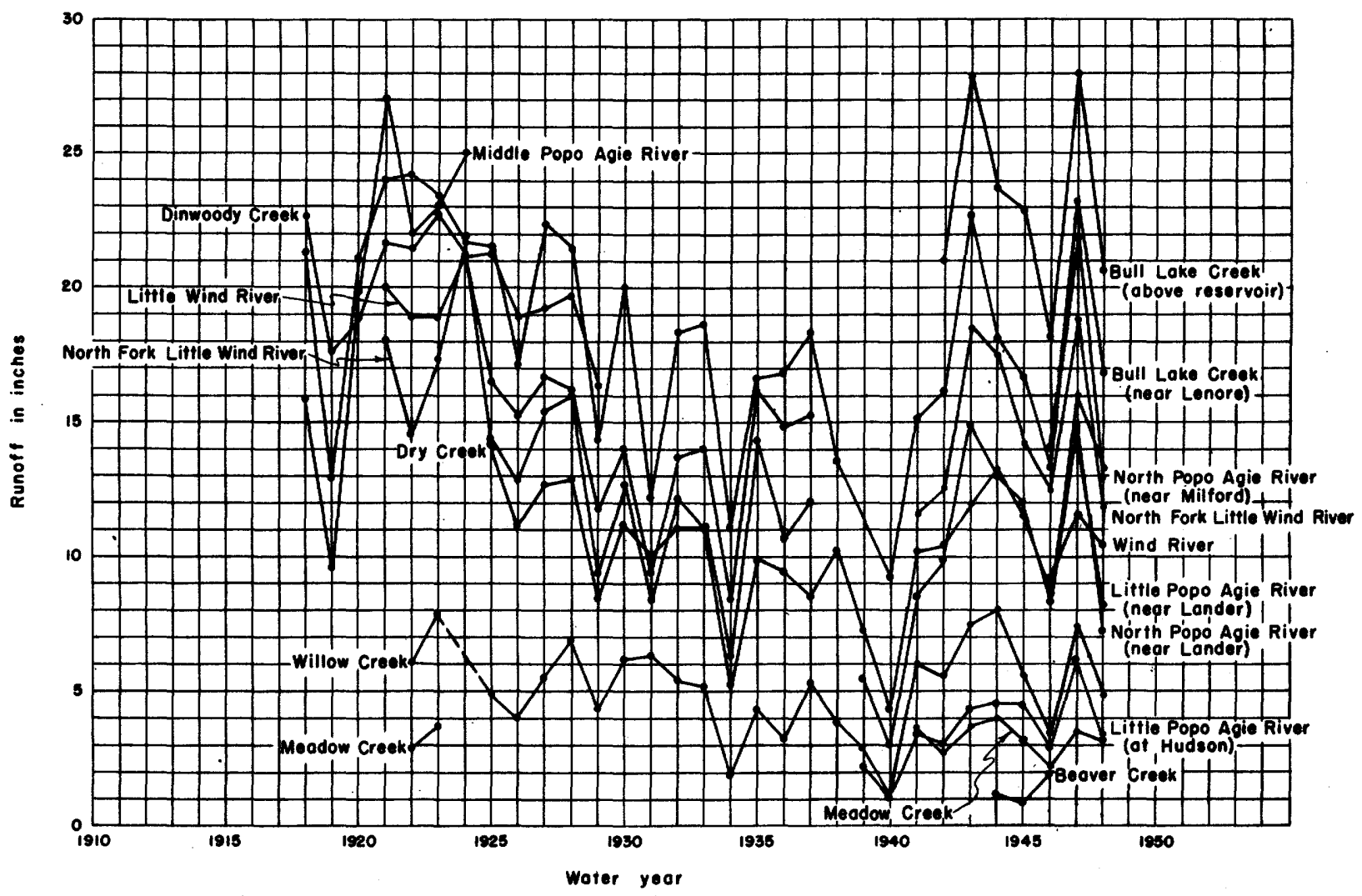

Figure 1.--Comporison of runoff measured of points olong Wind River Range.

\section{PRECIPITATION DATA}

Table 2 contains a list of the water-year precipitation totals for stations in or near the basin, as determined from publications of the U. S. Weather Bureau. I/ The periods of record are scattered at some of the stations and no attempt has been made to complete missing years by estimate. Colum two of table 2 lists the ratio, in percent, of the Lander annual water-year preclpitation to the average annual for the period 1918-48. These ratios were used as a guide in estimating the average annual munoff for the two stations on Badwater Creek. The precipitation data listed in table 2 were helpful in estimating the average annual muoff from the arid plains in the basin.

Records of snow stake readings, taken by the Weather Bureau at points in the mountains of the Wind River Range for the perlod 191541 , are published, $2 /$ as are records of snow surveys taken on a cooperative basis by Federal and State agencies from 1936 to

1 U. S. Department of Commerce, Weather Bureau, Climatological Data, Woming.

2 Idem.
1949.3/ The data from the snow stake and snow surve $\bar{y}$ determinations are valuable as a guide in estimating the precipitation at high altitude along the Wind River Range. Table 3 contains a summary of the snow stake and snow survey data available in the basin.

\section{Areal Variation of Precipitation}

Four correlations were made to determine the degree of representation of precipitation data for one point in estimating the precipitation on the surrounding area. The base station used was Lander and correlations of annual precipitation were made with stations at Dubols, Middle Fork, Riverton, and Ervay with resulting coefficients of correlation of $0.34,0.60,0.81$, and 0.70 respectively. These results may be interpreted to indicate that annual precipitation, measured at a point in the arid plains region of the basin, may be representative of a sizeable area adjacent to that point, but that there is relatively poor relationship between plains precipitation and foothill precipitation.

3 U. S. Department of Agriculture, Soll Conservation Service and Colorado Agricultural Experiment Station, Federal-State Cooperative Snow Surveys and Irrigation Water Forecasts for Missouri-Arkansas Drainage Basin. 
Tahle 2.-Inoual precipitation records of stations in or adjacent to Wind River Basin

\begin{tabular}{|c|c|c|c|c|c|c|c|c|c|c|c|}
\hline \multirow{2}{*}{$\begin{array}{l}\text { Water } \\
\text { Year }\end{array}$} & \multirow{2}{*}{$\begin{array}{l}\text { Ratio of Lander } \\
\text { precipitation to } \\
\text { Lander average } \\
\text { (percent) }\end{array}$} & \multicolumn{10}{|c|}{ Precipitation in inches } \\
\hline & & Diversion Dan & Dubois & Brrat & Fort Washaikie & Lander & Pevillion & Riverton & Salt Creek & Thermopolis & Middle Fork \\
\hline $\begin{array}{l}1918 \\
1919 \\
1920\end{array}$ & $\begin{array}{r}93 \\
53 \\
127\end{array}$ & $\bar{z}$ & $\begin{array}{c}10.24 \\
=\end{array}$ & $\begin{array}{l}15.20 \\
10.16 \\
18.85\end{array}$ & $=$ & $\begin{array}{r}13.05 \\
7.47 \\
17.88\end{array}$ & $\underline{-}$ & 7.39 & $=$ & $\overline{-}$ & $\begin{array}{c}18.68 \\
10.58 \\
-\end{array}$ \\
\hline $\begin{array}{l}1921 \\
1922 \\
1923 \\
1924 \\
1925\end{array}$ & $\begin{array}{r}138 \\
83 \\
141 \\
218 \\
60\end{array}$ & $\begin{array}{r}11.98 \\
6.70 \\
13.09 \\
9.93 \\
8.87\end{array}$ & $\begin{array}{c}- \\
\overline{-} \\
7.22 \\
8.15\end{array}$ & $\begin{array}{l}15.17 \\
23.43 \\
14.71 \\
19.26\end{array}$ & $\begin{array}{l}\overrightarrow{-} \\
\overrightarrow{-}\end{array}$ & $\begin{array}{r}19.58 \\
11.61 \\
19.90 \\
16.72 \\
8.46\end{array}$ & $\begin{array}{c}- \\
= \\
9.69 \\
9.14\end{array}$ & $\begin{array}{c}- \\
17.25 \\
9.83 \\
9.94\end{array}$ & $\begin{array}{c}- \\
- \\
17.23 \\
9.89 \\
12.65\end{array}$ & $\begin{array}{c}- \\
- \\
23.94 \\
13.88 \\
12.62\end{array}$ & $\begin{array}{c}\overline{-} \\
\overline{-} \\
19.12 \\
11.65\end{array}$ \\
\hline $\begin{array}{l}1926 \\
1927 \\
1928 \\
1929 \\
1930\end{array}$ & $\begin{array}{r}92 \\
104 \\
72 \\
122 \\
124\end{array}$ & $\begin{array}{r}5.75 \\
11.25 \\
8.29 \\
10.33 \\
12.73\end{array}$ & $\begin{array}{c}8.24 \\
- \\
7.88 \\
5.09 \\
9.70\end{array}$ & $\begin{array}{l}19.03 \\
17.61 \\
13.35 \\
19.57 \\
22.82\end{array}$ & $\begin{array}{c}\overline{-} \\
= \\
17.10\end{array}$ & $\begin{array}{l}12.90 \\
11.72 \\
10.12 \\
17.24 \\
17.58\end{array}$ & $\begin{array}{r}8.21 \\
12.04 \\
5.70 \\
7.01 \\
13.12\end{array}$ & $\begin{array}{r}10.65 \\
10.21 \\
7.39 \\
9.30 \\
10.30\end{array}$ & $\begin{array}{l}14.95 \\
15.55 \\
13.42 \\
19.24 \\
14.92\end{array}$ & $\begin{array}{c}17.84 \\
15.67 \\
11.39 \\
=\end{array}$ & $\begin{array}{l}20.53 \\
17.13 \\
15.19 \\
23.61 \\
20.89\end{array}$ \\
\hline $\begin{array}{l}1931 \\
1932 \\
1933 \\
1934 \\
1935\end{array}$ & $\begin{array}{r}92 \\
62 \\
107 \\
77 \\
99\end{array}$ & $\begin{array}{r}10.62 \\
6.88 \\
8.90 \\
6.31 \\
9.31\end{array}$ & $\begin{array}{l}7.47 \\
9.20 \\
7.89 \\
8.46 \\
6.49\end{array}$ & $\begin{array}{l}16.58 \\
12.47 \\
17.29 \\
10.85 \\
14.00\end{array}$ & $\begin{array}{r}14.76 \\
10.24 \\
12.49 \\
9.77 \\
12.57\end{array}$ & $\begin{array}{r}12.97 \\
8.78 \\
15.13 \\
10.89 \\
13.98\end{array}$ & $\begin{array}{l}7.72 \\
5.23 \\
9.16 \\
6.35 \\
6.86\end{array}$ & $\begin{array}{l}7.62 \\
6.20 \\
9.86 \\
6.59 \\
8.97\end{array}$ & $\begin{array}{l}13.85 \\
14.43 \\
18.39 \\
10.16 \\
14.38\end{array}$ & $\begin{array}{r}9.66 \\
10.76 \\
12.29 \\
7.25 \\
8.03\end{array}$ & $\begin{array}{l}15.39 \\
13.21 \\
18.28 \\
12.72 \\
18.88\end{array}$ \\
\hline $\begin{array}{l}1936 \\
1937 \\
1938 \\
1939 \\
1940\end{array}$ & $\begin{array}{r}83 \\
121 \\
84 \\
79 \\
75\end{array}$ & $\begin{array}{l}8.45 \\
9.21 \\
8.73 \\
7.77 \\
9.70\end{array}$ & $\begin{array}{l}7.87 \\
8.33 \\
9.65 \\
8.34 \\
8.56\end{array}$ & $\begin{array}{l}15.01 \\
23.24 \\
16.11 \\
14.16 \\
16.20\end{array}$ & $\begin{array}{r}11.55 \\
14.33 \\
9.85 \\
10.41 \\
9.97\end{array}$ & $\begin{array}{l}17.74 \\
17.08 \\
11.80 \\
11.04 \\
10.51\end{array}$ & $\begin{array}{l}6.42 \\
9.72 \\
8.68 \\
8.45 \\
7.82\end{array}$ & $\begin{array}{r}7.24 \\
10.97 \\
9.38 \\
8.10 \\
7.82\end{array}$ & $\begin{array}{r}7.63 \\
15.10 \\
11.67 \\
- \\
-\end{array}$ & $\begin{array}{r}6.37 \\
15.32 \\
12.80 \\
9.17 \\
10.93\end{array}$ & $\begin{array}{l}18.90 \\
22.00 \\
17.44 \\
15.02 \\
15.68\end{array}$ \\
\hline $\begin{array}{l}1941 \\
1942 \\
1943 \\
1944 \\
1945\end{array}$ & $\begin{array}{l}134 \\
102 \\
104 \\
138 \\
112\end{array}$ & $\begin{array}{r}12.33 \\
8.30 \\
7.22 \\
14.75 \\
13.10\end{array}$ & $\begin{array}{c}15.28 \\
9.68 \\
= \\
15.09\end{array}$ & $\begin{array}{c}22.96 \\
17.43 \\
= \\
=\end{array}$ & $\begin{array}{l}15.54 \\
12.14 \\
10.38 \\
17.32 \\
13.56\end{array}$ & $\begin{array}{l}18.94 \\
14.47 \\
14.66 \\
19.57 \\
15.75\end{array}$ & $\begin{array}{r}12.20 \\
8.73 \\
7.12 \\
15.04 \\
14.07\end{array}$ & $\begin{array}{r}13.21 \\
9.99 \\
10.60 \\
12.07 \\
11.78\end{array}$ & $\begin{array}{l}- \\
= \\
-\end{array}$ & $\begin{array}{c}16.95 \\
= \\
= \\
=\end{array}$ & $\begin{array}{l}23.94 \\
16.53 \\
19.44 \\
22.42 \\
20.36\end{array}$ \\
\hline $\begin{array}{l}1946 \\
1947 \\
1948\end{array}$ & $\begin{array}{r}82 \\
141 \\
83\end{array}$ & $\begin{array}{r}8.87 \\
15.54 \\
10.75\end{array}$ & $\begin{array}{r}8.89 \\
11.37 \\
8.82\end{array}$ & $=$ & $\begin{array}{l}10.47 \\
19.03 \\
10.38\end{array}$ & $\begin{array}{l}11.50 \\
19.91 \\
11.76\end{array}$ & $\begin{array}{r}9.57 \\
u_{4.38} \\
8.58\end{array}$ & $\begin{array}{r}9.09 \\
12.77 \\
7.48\end{array}$ & $\overline{-}$ & $\begin{array}{l}10.78 \\
14.74 \\
16.91\end{array}$ & $\begin{array}{l}17.21 \\
29.63 \\
16.60\end{array}$ \\
\hline
\end{tabular}


Table 3.--Snow stake and snow survey data avallable for Wind River Basin

\begin{tabular}{|c|c|c|c|c|c|c|}
\hline \multirow[b]{2}{*}{$\begin{array}{l}\text { Stake number } \\
\text { or survey name }\end{array}$} & \multirow[b]{2}{*}{$\begin{array}{l}\text { Elevation } \\
\text { (feet) }\end{array}$} & \multirow[b]{2}{*}{$\begin{array}{l}\text { Perlod of } \\
\text { record }\end{array}$} & \multicolumn{3}{|c|}{ Location } & \multirow{2}{*}{$\begin{array}{c}\text { Average seasonal water } \\
\text { content at end of March } \\
\text { (Inches) }\end{array}$} \\
\hline & & & Section & $\begin{array}{l}\text { Town- } \\
\text { sh1p }\end{array}$ & Range & \\
\hline $\begin{array}{l}325 \\
326 \\
327 \\
328 \\
349\end{array}$ & $\begin{array}{r}10,400 \\
8,900 \\
7,600 \\
6,900 \\
9,600\end{array}$ & & $\begin{array}{r}15 \\
7 \\
34 \\
23 \\
11\end{array}$ & $\begin{array}{l}31 N \\
31 N \\
32 N \\
32 N \\
43 N\end{array}$ & $\begin{array}{l}102 W \\
101 w \\
101 W \\
101 W \\
107 w\end{array}$ & $\begin{array}{r}11.67 \\
5.46 \\
6.25 \\
2.16 \\
10.69\end{array}$ \\
\hline $\begin{array}{l}350 \\
351 \\
352 \\
353 \\
354\end{array}$ & $\begin{array}{r}9,000 \\
8,500 \\
8,100 \\
10,000 \\
8,800\end{array}$ & & $\begin{array}{l}11 \\
27 \\
24 \\
14 \\
18\end{array}$ & $\begin{array}{l}43 \mathrm{~N} \\
43 \mathrm{~N} \\
43 \mathrm{~N} \\
4 \mathrm{~N} \\
41 \mathrm{~N}\end{array}$ & $\begin{array}{l}107 w \\
107 w \\
107 w \\
108 w \\
107 w\end{array}$ & $\begin{array}{r}9.53 \\
7.41 \\
5.81 \\
10.63 \\
8.46\end{array}$ \\
\hline $\begin{array}{l}355 \\
356 \\
357 \\
358 \\
359\end{array}$ & $\begin{array}{l}8,600 \\
8,500 \\
9,100 \\
7,750 \\
8,500\end{array}$ & & $\begin{array}{r}16 \\
6 \\
24 \\
3 \\
4\end{array}$ & $\begin{array}{l}41 N \\
41 N \\
44 N \\
42 N \\
42 N\end{array}$ & $\begin{array}{l}107 w \\
107 w \\
110 w \\
109 w \\
109 w\end{array}$ & $\begin{array}{r}8.01 \\
6.89 \\
18.69 \\
9.08 \\
9.83\end{array}$ \\
\hline $\begin{array}{l}\text { Brooks Lake } \\
\text { Blue Ridge } \\
\text { Du Noir } \\
\text { Mosquito Park Ranger Sta. }\end{array}$ & $\begin{array}{l}8,900 \\
9,200 \\
9,500 \\
8,750 \\
9,500\end{array}$ & $\begin{array}{c}1936-47 \\
1940-47 \\
1941-47 \\
1941-44,46,47\end{array}$ & $\begin{array}{r}8 \\
23 \\
23 \\
27 \\
23\end{array}$ & $\begin{array}{l}42 N \\
44 N \\
31 N \\
42 N \\
25 N\end{array}$ & $\begin{array}{r}109 w \\
110 w \\
101 w \\
108 w \\
3 w\end{array}$ & $\begin{array}{c}9.42 \\
19.7 \\
9.3 \\
9.5 \\
8.1\end{array}$ \\
\hline $\begin{array}{l}\text { St. Lawrence Ranger Station } \\
\text { Sawmlll Glade } \\
\text { Sherldan Creek Ranger Sta. } \\
\text { South Pass } \\
\text { T-Cross Ranch }\end{array}$ & $\begin{array}{l}9,000 \\
8,500 \\
7,500 \\
9,000 \\
8,000\end{array}$ & $\begin{array}{c}1941-44,47 \\
1940-47 \\
1936-47 \\
1940-47 \\
1941-47\end{array}$ & $\begin{array}{r}26 \\
3 \\
3 \\
13 \\
1\end{array}$ & $\begin{array}{l}1 \mathrm{~N} \\
31 \mathrm{~N} \\
42 \mathrm{~N} \\
30 \mathrm{~N} \\
43 \mathrm{~N}\end{array}$ & $\begin{array}{r}4 W \\
101 W \\
109 W \\
101 W \\
107 W\end{array}$ & $\begin{array}{r}7.4 \\
5.4 \\
6.0 \\
11.8 \\
6.4\end{array}$ \\
\hline
\end{tabular}

\section{Varlation of Precipitation with Altitude}

The annual munoff from drainage basins on the slopes of the high mountains bordering the basin is largely dependent on the annual. precipitation falling upon these basins. No records of annual precipitation for the regions of medium and high altitude within the bas in are avallable; therefore, it was necessary to compute the probable precipitation at these altitudes from all avaliable information. The following paragraphs of this section contain the computation procedure used in the determination of the amount of annual precipitation at altitudes along the Wind kiver Range.

Figure 2, a plot of the average annual precipitation for stations Lander, Riverton, and Middle Fork (for the period of continuous record, 1824-48) against altitude in feet above sea-level, shows the rapid increase of annua? precipitation with increase in altitude. Three snow surveys just south of Middle Fork Neather station have been used in estimating the precipitation at altitudes between 8,500 feet and 9,500 feet to extend the graph to the higher altitudes. They are: Sawmill Glade, Blue Ridge, and South Pass. The method of extension used is described in the following discussion.

Consideration of the monthly mean temperatures at Middle Fork indicated the months during which snow melt would not likely occur. The Middle Fork precipitation was totaled for the period from November 1 until the end of the spring month just before the. month of rapid snow melt. This gave a figure of the accumlated precipitation at
Middle Fork that could ve compared with the pesults of the snow surveys. The assumption was made (based upon temperature studies and empirical evaporation formulas) that evaporation from the snow at the higher altitudes was negligible during the winter months. Data for the comparison are listed in table 4. The water content value from the snow survey in each year was divided by the Middle Fork corresponding accumulated precipitation to give a ratio, expressed as a percentage, for the increase of precipitation with altitude for the winter months. The assumption mas made that this ratio computed for an approximate period of a halfyear holds true for an entire year, on an average. The ratios were averaged for the perlod 1941-48, and the result was used to compute an average annual precipitation for the altitude of the snow survey based on the average annual Middle Fork precipitation for the period 1924-48. These values are plotted on figure 2 . The relationship, determined for the section across the wind River Range and passing through Riverton and Lander, results in an annual precipitation of about 50 inches at an altitude of 12,000 feet. The slope of the line of relationship gives an increase of precipltation of 6 inches per 1,000 feet increase in altiEude. The relationship of precipltation and altitude determined for the section Riverton-Lander-Middle Fork and up to the crest of the divide is applicable only for that immediate vicinity.

An investigation of other areas of mountainous terrain was made to determine if there were some groupings of Weather Bureau stations that would give a series of observations, starting in the valley floor and extending up the mountain slope to a point near the crest. 


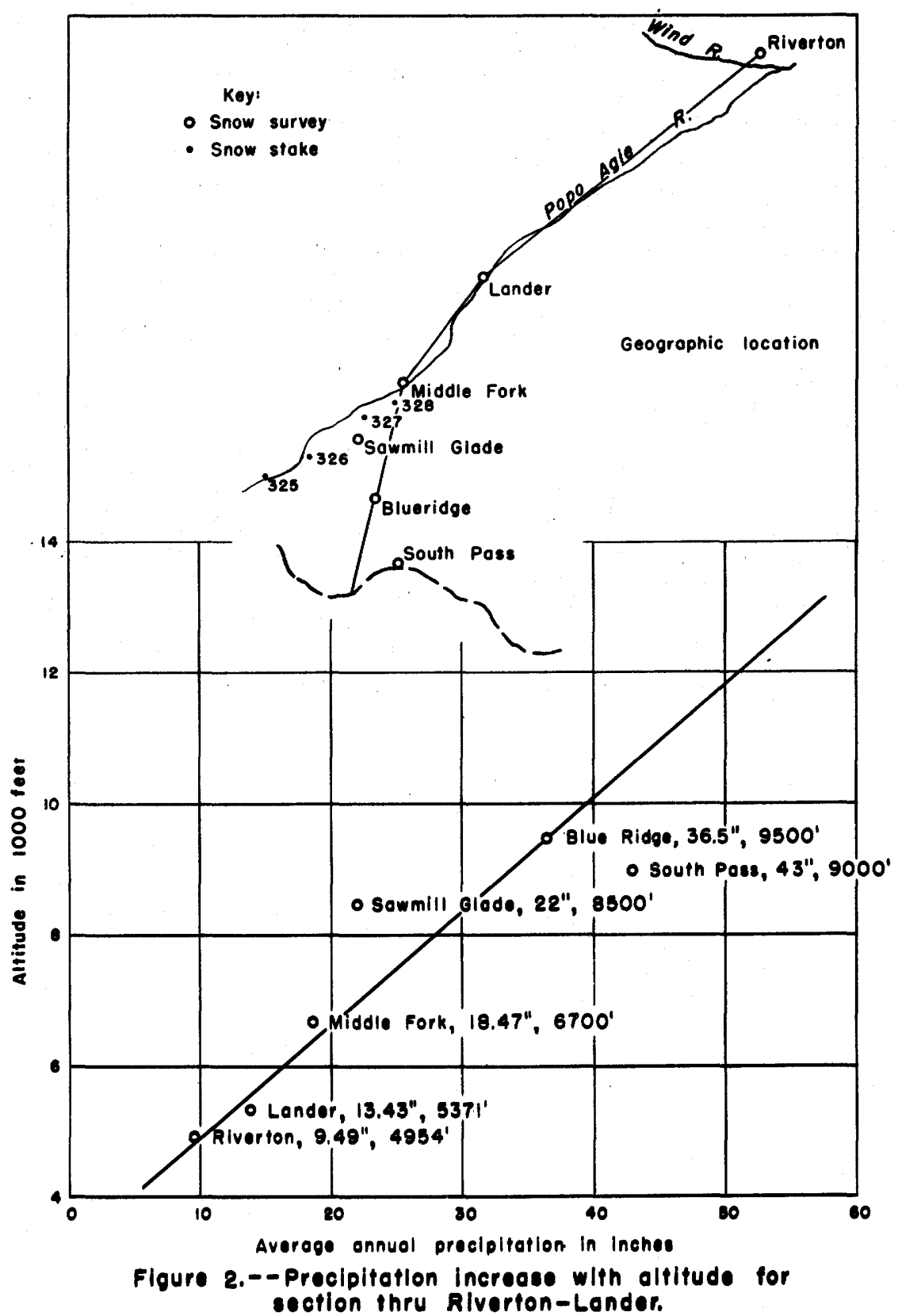

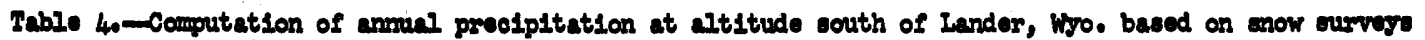

\begin{tabular}{|c|c|c|c|c|c|c|c|c|c|c|c|c|c|}
\hline \multirow[b]{2}{*}{$\begin{array}{l}\text { Water } \\
\text { jear }\end{array}$} & \multicolumn{7}{|c|}{ Muddle rork Proolpitation } & \multirow{2}{*}{$\begin{array}{l}\text { Blue } \\
\text { Ridge } \\
\text { water } \\
\text { oontent, } \\
\text { (Inchos) }\end{array}$} & \multirow{2}{*}{$\begin{array}{l}\text { South } \\
\text { Peas } \\
\text { mater } \\
\text { oontent, } \\
\text { (Inohed) }\end{array}$} & \multirow{2}{*}{$\begin{array}{l}\text { Sanemill } \\
\text { Glade } \\
\text { water } \\
\text { content, } \\
\text { (Inohes) }\end{array}$} & \multicolumn{3}{|c|}{ Percent of Meddle Fork } \\
\hline & Nov & Deo & Jen & Fob & Mar & Apr & Sum & & & & $\begin{array}{l}\text { Blue } \\
\text { RIdge }\end{array}$ & $\begin{array}{l}\text { South } \\
\text { Pass }\end{array}$ & $\begin{array}{l}\text { Sanmill] } \\
\text { GIade }\end{array}$ \\
\hline $\begin{array}{l}1941 \\
1942 \\
1943 \\
1944 \\
1945\end{array}$ & $\begin{array}{l}2.01 \\
0.43 \\
2.55 \\
0.59 \\
1.52\end{array}$ & $\begin{array}{l}0.83 \\
0.92 \\
0.08 \\
1.50 \\
0.55\end{array}$ & $\begin{array}{l}0.02 \\
0.75 \\
2.85 \\
1.03 \\
0.54\end{array}$ & $\begin{array}{l}0.62 \\
0.92 \\
0.21 \\
1.11 \\
0.60\end{array}$ & $\begin{array}{l}2.58 \\
1.04 \\
1.13 \\
2.65 \\
1.57\end{array}$ & $\begin{array}{l}6.01 \\
6.31 \\
5.60\end{array}$ & $\begin{array}{r}12.07 \\
4.06 \\
6.82 \\
13.19 \\
10.38\end{array}$ & $\begin{array}{r}13.8 \\
7.1 \\
17.1 \\
19.3 \\
17.4\end{array}$ & $\begin{array}{r}14.0 \\
8.7 \\
19.9 \\
17.0 \\
18.1\end{array}$ & $\begin{array}{r}10.9 \\
5.2 \\
9.3 \\
15.0 \\
13.5\end{array}$ & $\begin{array}{l}114 \\
175 \\
250 \\
116 \\
168\end{array}$ & $\begin{array}{l}116 \\
215 \\
292 \\
129 \\
174\end{array}$ & $\begin{array}{r}91 \\
128 \\
136 \\
114 \\
130\end{array}$ \\
\hline $\begin{array}{l}1946 \\
1947 \\
1948\end{array}$ & $\begin{array}{l}1.43 \\
1.23 \\
1.38\end{array}$ & $\begin{array}{l}0.44 \\
0.18 \\
0.84\end{array}$ & $\begin{array}{l}0.44 \\
0.36 \\
1.59\end{array}$ & $\begin{array}{l}0.18 \\
0.59 \\
1.01\end{array}$ & $\begin{array}{l}1.52 \\
1.18\end{array}$ & & $\begin{array}{l}4.02 \\
3.54 \\
4.82\end{array}$ & $\begin{array}{r}9.0 \\
11.2 \\
9.3\end{array}$ & $\begin{array}{r}13.8 \\
13.9 \\
9.2\end{array}$ & $\begin{array}{l}4.1 \\
4.5 \\
6.0\end{array}$ & $\begin{array}{l}225 \\
316 \\
193\end{array}$ & $\begin{array}{l}345 \\
393 \\
191\end{array}$ & $\begin{array}{l}102 \\
127 \\
125\end{array}$ \\
\hline \multicolumn{11}{|c|}{ Average percent, $1941-48$} & 198 & 232 & 119 \\
\hline \multicolumn{11}{|c|}{ Computed precipitation besed on MLddle Fork preolpitation 1924-48 } & $36.5^{n}$ & $43.0^{n}$ & 22.011 \\
\hline
\end{tabular}


Such a group was found in Idaho: Kellog, altitude 2,305 feet; Avery, altitude 2,500 feet; Wallace, altitude 2,770 feet; and Roland, altitude 4,150 feet. These station-average annual precipitations, plotted against altitude, give a linear relationship with a slope of 12 inches precipitation increase per 1,000 feet increase in altitude. Lee 4/found an ind1cated increase of 5 inches per 1,000 feet in the mountains west of Owens Valley in California with a linear relationship between precipitation and altitude.

The foregoing facts are mentioned to indicate that substantial evidence exists to warrant the assumption of linear increase of precipitation with increasing altitude, and that the 50-inch precipitation computed for 12,000 feet has some verification.

A study based on Ft. Washakle precipitation records and snow surveys gave a similar increase of precipitation with increasing altitude as for the section Lander-Middle Fork, and a study made for the western end of the basin based on Dubois and T-Cross Ranch preclpitation data and snow surveys indicates that the precipitation increase with altitude is nearly 12 inches per 1,000 feet. The above graphs of precipitation variation with altitude are combined for comparison on figure 3 .

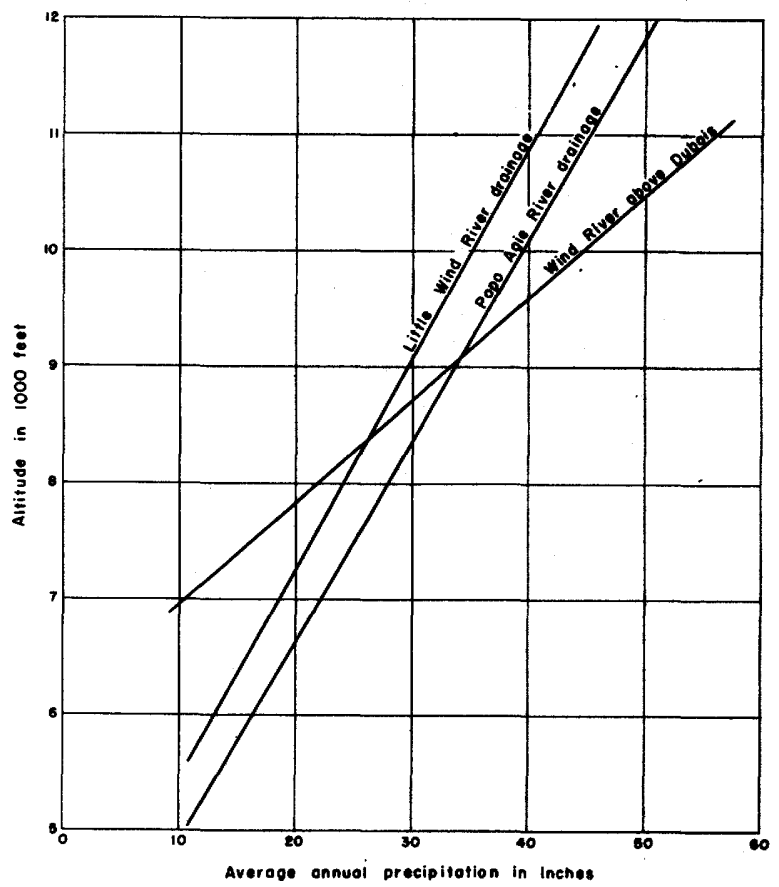

Figure 3.--Comparison of precipitation-altitude graphs for sections of Wind River Ronge.

\section{AVERAGE ANNUAL WATER LOSS}

Figure 4 presents a graph of the variation

4 Lee, C.H., An intensive study of the water resources of a part of Owens valley, California: Water-supply Paper 294, pl. 8, 1912 . of the average annual water loss with altitude for the Wind River Range north slope. The graph is not applicable for the south slopes of the Owl Creek Mountains, as the losses are greater there because of the more direct exposire to sunshine. The loss varlation graph was determined from the precipitation obtained from the previously described precipitationaltitude graphs (using the value of precipitation corresponding to the mean drainage basin altitude) and the measured runoff at the gage. The Ioss-altitude graph is a composite curve, drawn through the plotted points, determined in that manner for all the streams of the north slope of the Wind River Range.

\section{VARIATION OF RUNOFF WITH ALTITUDE}

The previously discussed precipitationaltitude graphs are linear above 9,000 feet; therefore, runoff, the residual, varies as a straight ine with altitude above that elevation. This relationship is true if the assumptions outlined in the previous discussion of the variation of precipitation with altitude are tmue. In this study the runoff has been considered to vary directly and linearly with altitude above elevations of 8,500 feet. On this basis topography determines the spacing of the 18ograms for areas of high altitude.

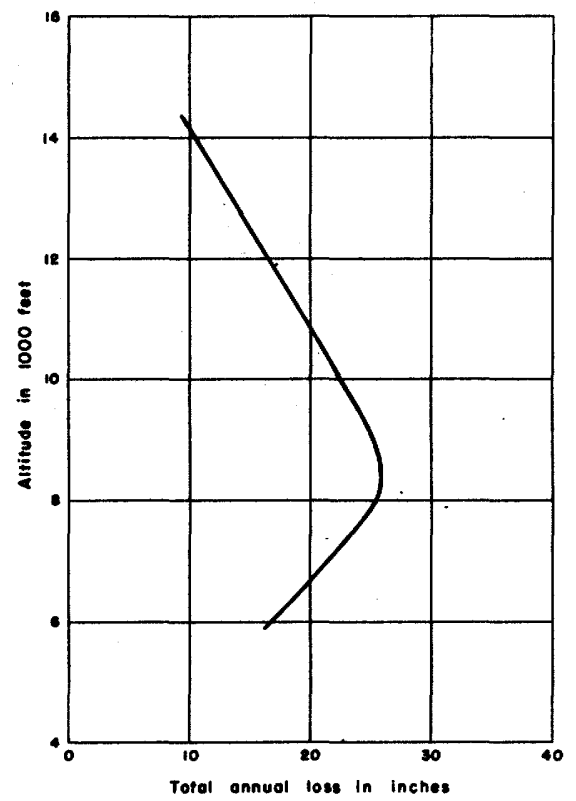

Figure --Composite averoos onnual lose variation with oltitude olong north. side Wind River Ronge.

TOPOGRAPHY

A map of the 1,000-foot contours of elevation above sea-level was prepared for this study to ald in the spacing of the isograms. Data for construction of the contours were obtained from Geological Survey quadrangle maps, the Riverton Project Map,6 the Casper sheet of World Aeronautical Charts, and an aerial mosiac. The contour map is only approximately accurate for the areas not covered by

6 U. S. Department of Interior, Bureau of Reclariction, Riverton Irrigation Project, 1939 . 
regular quadrangle maps, "but it is of sufficlent accuracy for this study. The contour map of the basin is presented on plate 2 .

\section{PLOTTING OF ISOGRAMS}

The isograms plotted along the slopes of the Wind River Range were spaced according to the topography with the quantitative amount determined by the precipitation-altitude and lossaltitude graphs applicable for the region. The results were checked against measured runoff on individual drainage areas. The slope drainage from the Owl Creek Mountains into the Wind River Basin has not been gaged; isograms were located along this region in quantitative amount by estimate based on the muoff from South Fork Owl Creek, and the spacing was governed by topography. Isograms for the lowaltitude, arld part of the basin were estimated on the basis of a few scattered records, by a fleld reconnalssance, and by the ald of topography and climatic data. The complete average annual runoff map for the Wind River Basin is shown on plate 1. Isograms of 0.25 , $0.50,1.0,3.0,5.0,10.0,15.0,20.0,30.0$, and 40.0 inches have been chosen for the plot, as they give a representative spacing. The area within the 0.25 inch isogram has average annual runoff less than 0.25 inch, but is belleved to have more than 0.10 inch average annual runoff generally.

\section{ACOURACY OF MAP}

A figure of average annual munoff computed for the drainage area above Boysen Dam by planimetering the areas bounded by the 1sograms on plate 1 agrees very well with the long-term average runoff measured at the Thermopolis gage after suitable irrigation diversion corrections are made. The runoff map is considered to be of good accuracy in expressing the average annual runoff from the mountains bordering the basin and of fair accuracy in the arid part of the basin. 


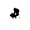

$\bullet$

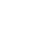
t.

a. 